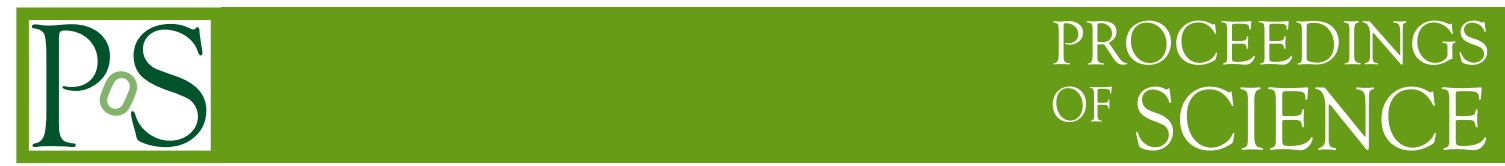

\title{
Search for exotic resonances with top quarks
}

\section{Davide Pagano* for the CMS Collaboration}

Université catholique de Louvain

E-mail: davide.pagano@cern.ch

A summary of the CMS Collaboration results on searches for new high-mass resonances decaying to top quarks, predicted in many scenarios beyond the Standard Model (BSM), is presented. Results were produced with data collected at the Large Hadron Collider at a center-of-mass energy of $7 \mathrm{TeV}$ and $8 \mathrm{TeV}$, with integrated luminosities ranging between 5.0 and $19.6 \mathrm{fb}^{-1}$. No evidence for such new resonances was observed and exclusion limits for many BSM models were set.

XXI International Workshop on Deep-Inelastic Scattering and Related Subject -DIS2013, 22-26 April 2013

Marseilles, France

\footnotetext{
${ }^{*}$ Speaker.
} 


\section{Introduction}

The top quark, because of its large mass, is expected to play a key role in electroweak symmetry breaking and be a privileged window on new physics. Many scenarios beyond the Standard Model (BSM) feature new gauge interactions with enhanced couplings to the third generation quarks. These couplings result in new heavy gauge bosons, generally indicated as $Z^{\prime}$ and $W^{\prime}$, which could manifest as resonances in the $t \bar{t}$ or $t b$ invariant mass distributions. Example of models predicting new particles, coupling predominantly to third generation quarks, include topcolor models [1], models with extra space-time dimensions as, for example, the Kaluza-Klein excitations of the SM gauge bosons [2], and little Higgs theories [3]. In addition to new high-mass gauge bosons decaying to top quarks, another interesting BSM scenario, having an impact on the top quark production, is the existence of excited $t$-quarks, $t^{*}$, decaying to a $t$-quark and a gluon [4].

This paper presents the results of CMS direct searches for $Z^{\prime}$ and $W^{\prime}$ resonances decaying to $t \bar{t}$ and $t b$ respectively, based on data collected in 2011 and 2012 at a center-of-mass energy of $7 \mathrm{TeV}$ and $8 \mathrm{TeV}$, between 5.0 and $19.6 \mathrm{fb}^{-1}$. Also the CMS search for pair-produced $t^{*}$, which is the first dedicated search for excited $t$-quarks, is presented. A detailed description of the CMS experiment is provided in Ref. [5], while the full descriptions of the analyses presented here can be found in Ref. $[6,7,8,9,10]$.

\section{Search for excited top quarks}

This section summarizes the CMS search for pair-produced excited $t$-quarks $\left(t^{*}\right)$, using 19.6 $f b^{-1}$ of data at $\sqrt{s}=8 \mathrm{TeV}[6]$. The analysis assumed a model with a spin $3 / 2 t^{*}$ quark, decaying exclusively to a $t$-quark and gluon, and considered events having a single lepton in the final state.

Events were requested to have exactly one isolated muon (electron) with transverse momentum $p_{T}>26$ (30) $\mathrm{GeV}$, at least six jets with $p_{T}>30 \mathrm{GeV}$, at least one $b$-tagged jet and transverse missing energy $E_{T}^{\text {miss }}>20 \mathrm{GeV}$. A shape analysis on the reconstructed mass of $t^{*}$ candidates $\left(M_{t^{*}}\right)$ was performed to look for the $t^{*} t^{*}$ signal. The resolution on $M_{t^{*}}$ was improved with a kinematic fit, constraining the momenta of the final state particles. No evidence of the signal was observed in data and exclusion limits were set. Figure 1 shows the expected and observed 95\% CL upper limits for the $t^{*} t^{*}$ production cross-section as a function of the $t^{*}$ mass. From the comparison of the results with the predicted cross-section in the spin-3/2 Randall-Sundrum model [11], masses below $790 \mathrm{GeV}$ were excluded.

\section{Search for $t b$ resonances}

In this section a summary of the CMS search for heavy gauge bosons $W^{\prime}$, decaying into a $t$ quark and a $b$-quark, is presented [7]. The analysis, based on $19.6 \mathrm{fb}^{-1}$ of data at $\sqrt{s}=8 \mathrm{TeV}$, was performed with events where the $t$-quark decayed leptonically. Arbitrary combination of left- $\left(a^{L}\right)$ and right-handed couplings $\left(a^{R}\right)$, included in the effective interaction Lagrangian of the $W^{\prime}$ boson to the SM fermions [12], was considered.

Events were requested to have one isolated muon or electron with $p_{T}>50 \mathrm{GeV}$, at least two jets with $p_{T}>120,40 \mathrm{GeV}$, at least one $b$-tagged jet and $E_{T}^{m i s s}>20 \mathrm{GeV}$. The analysis strategy 


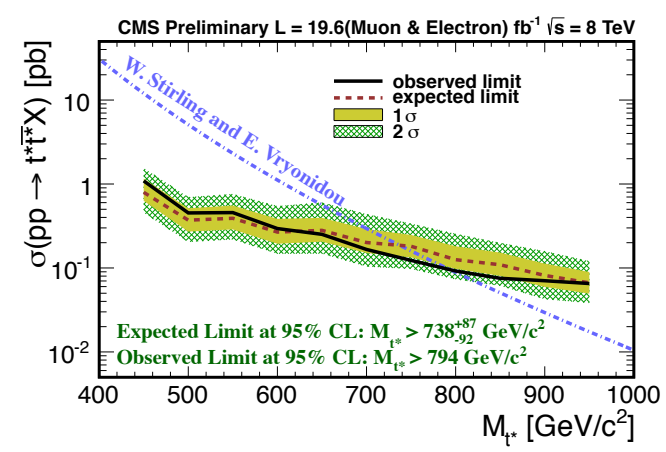

Figure 1: $t^{*}$ search - 95\% C.L. expected and observed limits on $\sigma t^{*} t^{*}$ as a function of $M_{t *}$ [6].

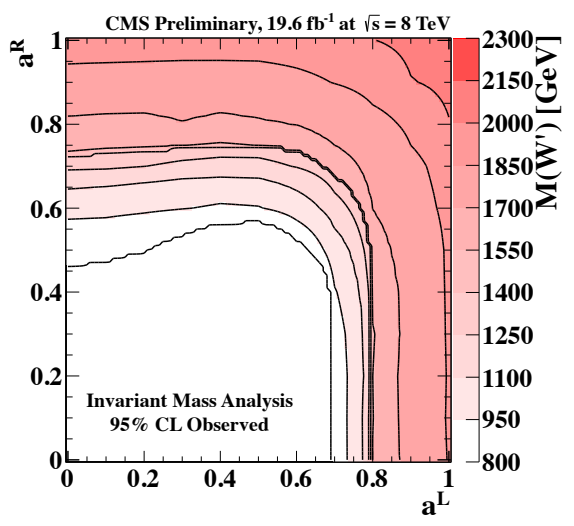

Figure 2: $W^{\prime} \rightarrow t b$ search - 95\% C.L. observed limits on $M_{W^{\prime}}$ as a function of $a^{L}$ and $a^{R}$ [7].

was based on a template fit to the reconstructed $t b$ invariant mass, using templates from the MC simulation. No evidence of the $W^{\prime}$ signal was found and upper limits on the signal production cross-section $\left(\sigma_{W^{\prime}}\right)$, as a function of the $W^{\prime}$ mass, were set. By parametrizing $\sigma_{W^{\prime}}$ in terms of $a^{L}$ and $a^{R}$ (see Ref. [7] for details), 95\% C.L. upper limits on the $W^{\prime}$ boson mass as a function of the left- and right-handed couplings to fermions were set, as shown in Figure 2. In the particular case of right-handed $W^{\prime}$ bosons $\left(a^{R}=1\right.$ and $\left.a^{L}=0\right)$ the observed (expected) limit was $2.03(2.09) \mathrm{TeV}$.

\section{Search for $t \bar{t}$ resonances in the fully hadronic channel}

This section presents a summary of the CMS search for high-mass resonances $\left(Z^{\prime}\right)$ decaying to top quark-antiquark pairs, using the full hadronic final state [8]. The search, based on $19.6 \mathrm{fb}^{-1}$ of data at $\sqrt{s}=8 \mathrm{TeV}$, was optimized to exploit the boosted nature of the $t$-quarks from the high-mass resonances decays, whose decay products tend to merge and be reconstructed as a single jet.

Two types of top quark candidates were considered: 'type 1' candidates, with decay products merged into a single jet, and 'type 2' candidates, containing a merged $W$ boson jet, but a separate additional jet corresponding to the $b$-quark. The analysis benefited from the use of a top tagging algorithm [13] for type 1 candidates, and the jet pruning algorithm [14] to identify boosted $W$ boson jets for type 2 candidates. Events were requested to have two type 1 candidates with $p T>$ $400 \mathrm{GeV}$ (type 1+1 channel). A control sample was selected by requiring one type 1 and one type 2 candidates (type $1+2$ channel). The search for new high-mass resonances was based on a template fit to the reconstructed $t \bar{t}$ invariant mass. No evidence of the signal was observed and exclusion limits for the RS KK gluon [15] and models with narrow $(\Gamma / m=1 \%)$ and wide $(\Gamma / m=$ $10 \%) Z^{\prime}$ resonances were set. Figure 3 shows the $95 \%$ expected and observed limits on the signal production cross-section, with the theoretical prediction for the RS KK gluon: masses below 1.8 $\mathrm{TeV}$ were excluded.

\section{Search for $t \bar{t}$ resonances in the fully leptonic channel}

A summary of the CMS search for narrow high-mass resonances $\left(Z^{\prime}\right)$ decaying to $t \bar{t}$ pairs, 


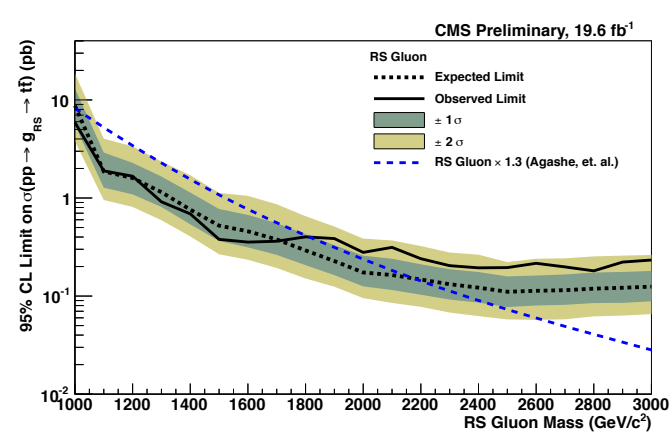

Figure 3: $Z^{\prime} \rightarrow t \bar{t}$ fully hadronic - 95\% C.L. expected and observed limits on the RS gluon production cross-section as function of the mass [8].

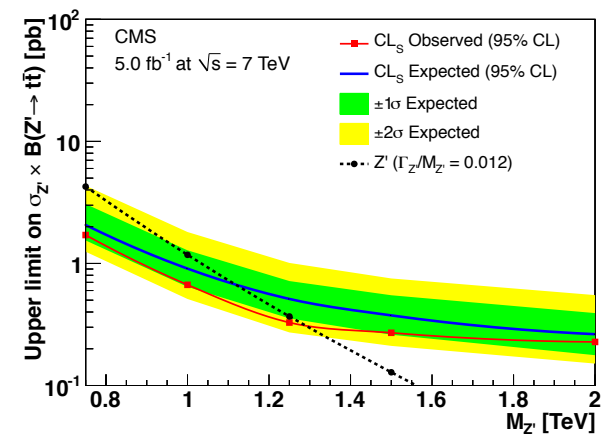

Figure 4: $Z^{\prime} \rightarrow t \bar{t}$ fully leptonic - 95\% C.L. upper limits on $\sigma_{Z^{\prime}} \times B$ versus $M_{Z^{\prime}}$ with the theoretical prediction for a leptophobic $Z^{\prime}$ [9].

using the fully leptonic final state, is presented in this section [9]. The analysis was performed using $5.0 \mathrm{fb}^{-1}$ of data at $\sqrt{\mathrm{s}}=7 \mathrm{TeV}$.

Events were requested to have two isolated leptons $(e e, e \mu$ and $\mu \mu)$ with $p_{T}>20 \mathrm{GeV}$, at least two jets with $p_{T}>30 \mathrm{GeV}$ and at least one $b$-tagged jet. Additionally, for the $e e$ and $\mu \mu$ channels, the transverse missing energy was requested to be greater than $30 \mathrm{GeV}$. The analysis adopted a multivariate approach, based on a Bayesian neural network (BNN). A set of 17 variables was used to train the BNN and the shapes of its outcoming outputs for the data, the SM background, and the signal were compared to test the presence of the signal. Good agreement between data and the SM background was found in all three channels and exclusion limits were set. Figure 4 shows the 95\% C.L. expected and the observed limits on the signal production cross-section times branching fraction, as a function of the $Z^{\prime}$ mass, with the theoretical prediction for a leptophobic $Z^{\prime}$ of width $\Gamma / m=1.2 \%$ [16]: masses below $1.3 \mathrm{TeV}$ were excluded.

\section{Search for $t \bar{t}$ resonances in the lepton plus jets channel}

This section summarizes the CMS search for heavy resonances decaying to $t \bar{t}$ pairs, using the lepton plus jets channel [10]. The analysis, based on $19.6 \mathrm{fb}^{-1}$ of data at $\sqrt{s}=8 \mathrm{TeV}$, benefited from the use of two different strategies: the threshold analysis, optimized to identify small boosted top quarks, and the boosted analysis, focusing on the search for resonance above $1 \mathrm{TeV}$, where highly boosted top quarks could result into partially or fully merged decay products.

Events in the threshold analysis were requested to have one isolated electron (muon) with $p_{T}$ $>30$ (26) $\mathrm{GeV}$, at least four jets with $p_{T}>70,50,30,30 \mathrm{GeV}$ and $E_{T}^{\text {miss }}>20 \mathrm{GeV}$. In the boosted analysis, events were required to have one electron (muon) with $p_{T}>35$ (45) $\mathrm{GeV}$ and without any requirement on the isolation, at least two jets with $p_{T}>150,50 \mathrm{GeV}, E_{T}^{\text {miss }}>50 \mathrm{GeV}$ and $H_{T}^{l e p}>150 \mathrm{GeV}$, being $H_{T}^{l e p}$ defined as the scalar sum of the lepton $p_{T}$ and $E_{T}^{\text {miss }}$. In both cases, a shape analysis was performed on the reconstructed $t \bar{t}$ invariant mass spectrum and the results were combined to set exclusion limits on the signal production cross-section. Figure 5 shows the $95 \%$ C.L. expected and observed limits on $\sigma_{Z^{\prime}} \times B$ as a function of the $Z^{\prime}$ mass, with the theoretical prediction for a $Z^{\prime}$ with $\Gamma / m=1.2 \%$ : masses below $2.1 \mathrm{TeV}$ were excluded. 


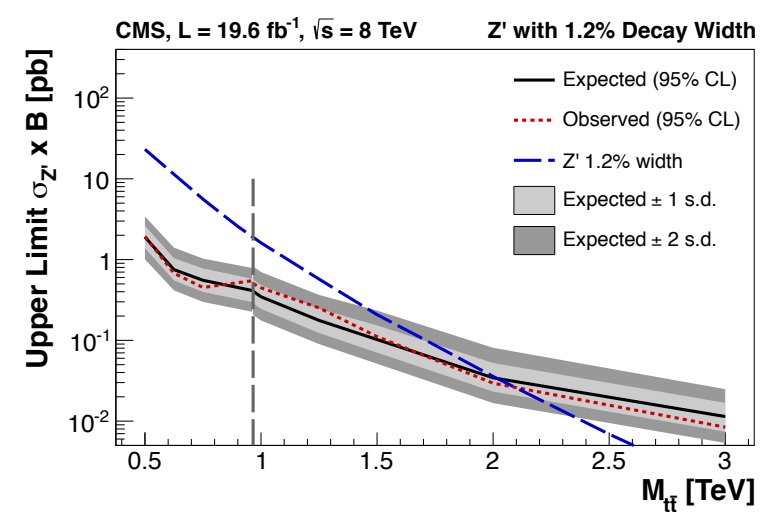

Figure 5: $Z^{\prime} \rightarrow t \bar{t}$ lepton + jets - 95\% C.L. expected and observed limits on the $\sigma_{Z^{\prime}} \times B$ as a function of the $Z^{\prime}$ mass. The theoretical prediction is for the $Z^{\prime}$ production with $\Gamma / m=1.2 \%$ [10].

\section{Conclusions}

An overview of the CMS searches for new high-mass resonances decaying to $t$-quarks, including the first direct search for excited top quarks, has been presented. These searches, adopting different final states and analysis strategies, were performed using $p-p$ collision data at $\sqrt{s}=7$ $\mathrm{TeV}$ and $8 \mathrm{TeV}$, with integrated luminosities ranging between 5.0 and $19.6 \mathrm{fb}^{-1}$. No evidence of such new resonances was observed and exclusion limits were set on several BSM scenarios.

\section{References}

[1] C. T. Hill, Phys. Lett. B 345, 483 (1995) [hep-ph/9411426].

[2] A. Djouadi, G. Moreau and R. K. Singh, Nucl. Phys. B 797, 1 (2008) [arXiv:0706.4191 [hep-ph]].

[3] M. Schmaltz and D. Tucker-Smith, Ann. Rev. Nucl. Part. Sci. 55, 229 (2005) [hep-ph/0502182].

[4] W. J. Stirling and E. Vryonidou, JHEP 1201, 055 (2012) [arXiv:1110.1565 [hep-ph]].

[5] S. Chatrchyan et al. [CMS Collaboration], JINST 3, S08004 (2008).

[6] S. Chatrchyan et al. [CMS Collaboration], CMS-PAS-B2G-12-014 (2013).

[7] S. Chatrchyan et al. [CMS Collaboration], CMS-PAS-B2G-12-010 (2013).

[8] S. Chatrchyan et al. [CMS Collaboration], CMS-PAS-B2G-12-005 (2013).

[9] S. Chatrchyan et al. [CMS Collaboration], Phys. Rev. D 87, 072002 (2013).

[10] S. Chatrchyan et al. [CMS Collaboration], CMS-PAS-B2G-12-006 (2013).

[11] L. Randall and R. Sundrum, Phys. Rev. Lett. 83, 3370 (1999) [hep-ph/9905221].

[12] Z. Sullivan, Phys. Rev. D 66, 075011 (2002) [hep-ph/0207290].

[13] D. E. Kaplan, K. Rehermann, M. D. Schwartz and B. Tweedie, Phys. Rev. Lett. 101, 142001 (2008).

[14] S. D. Ellis, C. K. Vermilion and J. R. Walsh, Phys. Rev. D 80, 051501 (2009).

[15] K. Agashe, A. Belyaev, T. Krupovnickas, G. Perez and J. Virzi, Phys. Rev. D 77 (2008) 015003

[16] R. M. Harris, C. T. Hill and S. J. Parke, hep-ph/9911288. 